\title{
A LOCAL LIMIT THEOREM FOR SUMS OF DEPENDENT RANDOM VARIABLES
}

\author{
Mei WANG and Michael WOODROOFE \\ Department of Statistics, The University of Michigan, Ann Arbor, MI 48109-1027, USA \\ Received July 1988 \\ Revised February 1989
}

\begin{abstract}
A local version of the central limit theorem is established for normalized sums of dependent random variables when a global theorem is known and conditional distributions are sufficiently smooth. The proof uses ideas from Statistics. by representing the density as the integral of a score function for a translation family of distributions.
\end{abstract}

AMS 1980 Subject Classification: $60 \mathrm{~F} 99$.

Keywords: Central limit theorem, almost differentiability, score function, martingales, stationary sequences, Markov chains.

\section{Introduction}

There is a substantial literature on the Central Limit Theorem for sums of dependent random variables, especially martingales and stationary sequences; several sets of sufficient conditions are known for the convergence in distribution of normed sums. See, for example, Hall and Heyde (1980, Chapters 3 and 5), the review paper by Peligrad (1986) and the references given there. There is much less work on local versions of the Central Limit Theorem for dependent sequences, however. Lalley (1986) notes the paucity of such work and proves a local theorem for Gibb's states.

The purpose of this note is to show that techniques developed by Klassen (1984), Boos (1985) and Sweeting (1986) may be used to establish local versions of the Central Limit Theorem for sums of dependent random variables, when a global theorem is known and conditional distributions are sufficiently smooth. The approach is suggested by the work of Jeganathan (1987).

\section{Preliminaries}

If $u \in \mathbb{R}^{n}$ and $f$ is a Borel measurable function from $\mathbb{R}^{n}$ into $\mathbb{R}$, then $f$ is said to be almost differentiable in the direction $u$ iff there is a measurable function $D f_{u}$ from $\mathbb{R}^{n}$ into $\mathbb{R}$ for which

$$
f(x \pm c u)-f(x)= \pm \int_{0}^{c} D f_{u}(x \pm t u) \mathrm{d} t
$$

for a.e. $x \in \mathbb{R}^{n}$ (Lebesgue) for each $c>0$. Here (1) includes the condition that the integral exists as a Lebesgue integral. It follows directly from Lemma 2, below, that the function $D f_{u}$ is essentially unique.

If $f$ is continuously differentiable, then (1) holds for all $x$ and $u$ with $D f_{u}(x)=u \cdot \nabla f(x)$, where $\nabla f$ denotes the gradient of $f$ and - denotes the dot product in $\mathbb{R}^{n}$; but (1) does not require continuous differentiability. Almost differentiability is used by Stein (1981). 
The following properties of almost differentiability are needed. The first of these is just the formula for integration by parts. The next two follow from routine applications of Fubini's theorem. The last then follows easily, since a derivative vanishes at a minimum.

Lemma 1. If $f$ and $g$ are almost differentiable in the direction $u \in \mathbb{R}^{n}$, then so is $f g$, and $D(f g)_{u}=f D g_{u}+g D F_{u}$.

Lemma 2. If $f$ is almost differentiable in the direction $u$, and if $f$ and $D f_{u}$ are integrable, then

$$
\lim _{t \rightarrow 0} \int_{\mathbb{R}^{n}}\left|\frac{f(x+t u)-f(x)}{t}-D f_{u}(x)\right| \mathrm{d} x=0 .
$$

Lemma 3. Suppose that $f$ is almost differentiable in the direction $u$ and that $f$ and $D f_{u}$ are integrable. Let

$$
\bar{f}\left(x_{1}, \ldots, x_{n-1}\right)=\int_{-\infty}^{\infty} f\left(x_{1}, \ldots, x_{n-1}, x_{n}\right) \mathrm{d} x_{n}
$$

for a.e. $\left(x_{1}, \ldots, x_{n-1}\right) \in \mathbb{R}^{n-1}$. Then $\bar{f}$ is almost differentiable in the direction $v=\left(u_{1}, \ldots, u_{n-1}\right)$, and

$$
D \vec{f}_{v}\left(x_{1}, \ldots, x_{n-1}\right)=\int_{-\infty}^{\infty} D f_{u}\left(x_{1}, \ldots, x_{n-1}, x_{n}\right) \mathrm{d} x_{n} \text { a.e. }
$$

Lemma 4. If $f \geqslant 0$ and $f$ is almost differentiable in the direction $u \in \mathbb{R}^{n}$, then $\left\{x \in \mathbb{R}^{n}: f(x)=0\right.$ and $\left.D f_{u}(x) \neq 0\right\}$ is a Lebesgue null set.

\section{Equivariant random variables}

Now let $X_{1}, \ldots, X_{n}$ denote jointly distributed random variables, defined on some probability space $(\Omega, \mathscr{A}, P)$; write $X-\left(X_{1}, \ldots, X_{n}\right)$ for the random vector; and suppose that $X$ has a density $f$ (with respect to $n$-dimensional Lebesgue measure). Let $\psi: \mathbb{R}^{n} \rightarrow \mathbb{R}$ be a Borel measurable, translation equivariant function; that is,

$$
\psi\left(x_{1}+b, \ldots, x_{n}+b\right)=\psi\left(x_{1}, \ldots, x_{n}\right)+c b
$$

for all $x=\left(x_{1}, \ldots, x_{n}\right)$ and $-\infty<b<\infty$ for some $0<c<\infty$, called the multiplier. Finally, let $Y$ denote a random variable of the form

$$
Y=\psi(X)=\psi\left(X_{1}, \ldots, X_{n}\right)
$$

and let

$$
H(y)=P\{Y \leqslant y\}, \quad-\infty<y<\infty,
$$

denote its distribution function.

Proposition 1. If $f$ is almost differentiable in the direction $1:=(1, \ldots, 1)$ and if $D f_{1}$ is integrable, then $H$ has density

$$
h(y)=\frac{1}{c} \int_{\{Y \leqslant y\}} l(X) \mathrm{d} P, \quad-\infty<y<\infty,
$$

where

$$
l(x)=\frac{D f_{1}(x)}{f(x)} I\{f(x)>0\}, \quad x \in \mathbb{R}^{n}
$$


Proof. That $E|l(X)|<\infty$ follows directly from the assumed integrability of $D f_{1}$. For any $y,-\infty<y<\infty$, and $t>0$,

$$
\begin{aligned}
H(y+t) & =\int_{\{x: \psi(x) \leqslant y+t\}} f\left(x_{1}, \ldots, x_{n}\right) \mathrm{d} x_{1} \cdots \mathrm{d} x_{n} \\
& =\int_{\{x: \psi(x) \leqslant y\}} f\left(x_{1}+\frac{t}{c}, \ldots, x_{n}+\frac{t}{c}\right) \mathrm{d} x_{1} \cdots \mathrm{d} x_{n},
\end{aligned}
$$

where the second equality follows from the change of variables $x_{i}=x_{i}^{\prime}+t / c$ for $i=1, \ldots, n$. Thus, in more compact notation,

$$
\begin{aligned}
\frac{H(y+t)-H(y)}{t} & =\int_{\{x: \psi(x) \leqslant y\}} \frac{1}{t}\left[f\left(x+\frac{t}{c}\right)-f(x)\right] \mathrm{d} x \\
& \rightarrow \int_{\{x: \psi(x) \leqslant y\}} \frac{1}{c} D f_{1}(x) \mathrm{d} x \\
& =\int_{\{x: \psi(x) \leqslant y\}} \frac{1}{c} l(x) f(x) \mathrm{d} x=\int_{\{Y \leqslant y\}} \frac{1}{c} l(X) \mathrm{d} P,
\end{aligned}
$$

as $t \rightarrow 0$ by Lemmas 2 and 4 . Thus, $H$ has a derivative at every $y$. It follows that $H$ is continuous and then that the right side of (3) is continuous. That the derivative of $H$ is its density follows easily.

\section{Normalized sums}

Let $X_{k}, k=1,2, \ldots$, denote a sequence of jointly distributed random variables, defined on a probability space $(\Omega, \mathscr{B}, P)$, for which $E\left(X_{k}\right)=0$ and $0<E\left(X_{k}^{2}\right)<\infty$ for all $k=1,2, \ldots$ Let

$$
S_{n}^{*}=\frac{1}{\sqrt{n}} \sum_{k=1}^{n} X_{k}
$$

and

$$
H_{n}(s)=P\left\{S_{n}^{*} \leqslant s\right\}, \quad s \in \mathbb{R}, n \geqslant 1 .
$$

Next, let $G_{k}$ be a regular conditional distribution function for $X_{k}$, given $X_{1}, \ldots, X_{k-1}$, that is

$$
G_{k}\left(x_{1}, \ldots, x_{k-1} ; x_{k}\right)=P\left\{X_{k} \leqslant x_{k} \mid X_{1}=x_{1}, \ldots, X_{k-1}=x_{k-1}\right\}
$$

for $-\infty<x_{1}, \ldots, x_{k}<\infty$ and $k=1,2, \ldots$; and suppose that $G_{k}$ has a continuous density $g_{k}$,

$$
g_{k}\left(x_{1}, \ldots, x_{k-1} ; x\right)=\frac{\partial G_{k}}{\partial x}\left(x_{1}, \ldots, x_{k-1} ; x\right) \quad \forall x, k \text {. }
$$

Then

$$
f_{n}\left(x_{1}, \ldots, x_{n}\right)=\prod_{k=1}^{n} g_{k}\left(x_{1}, \ldots, x_{k-1} ; x_{k}\right)
$$

defines a joint density for $X_{1}, \ldots, X_{n}$. Thus, if $f_{n}$ is almost differentiable in the direction 1 and $D f_{n, 1}:=D\left(f_{n}\right)_{1}$ is integrable, then $S_{n}^{*}$ has density

$$
h_{n}(s)=\frac{1}{\sqrt{n}} \int_{\left\{S_{n}^{*} \leqslant s\right\}} L_{n} \mathrm{~d} P, \quad s \in \mathbb{R}, n \geqslant 1,
$$


where

$$
L_{n}=l_{n}\left(X_{1}, \ldots, X_{n}\right), \quad n \geqslant 1,
$$

and $l_{n}$ is as in (2) with $f$ replaced by $f_{n}$.

Proposition 2. If $g_{k}$ is almost differentiable in the direction $1=(1, \ldots, 1)(k$ times $)$ for all $k \geqslant 1$, then $f_{n}$ is almost differentiable in the direction $1=(1, \ldots, 1)$ ( $n$ times) and

$$
\left(\frac{D f_{n, 1}}{f_{n}}\right)\left(x_{1}, \ldots, x_{n}\right)=\sum_{k=1}^{n}\left(\frac{D g_{k, 1}}{g_{k}}\right)\left(x_{1}, \ldots, x_{k}\right)
$$

a.e. on $\left\{x: f_{n}\left(x_{1}, \ldots, x_{n}\right)>0\right\} \subseteq \mathbb{R}^{n}$ for all $n=1,2, \ldots$ If , in addition,

$$
\tau_{k}^{2}:=E\left\{\left[\left(\frac{D g_{k, 1}}{g_{k}}\right)\left(X_{1}, \ldots, X_{k-1} ; X_{k}\right)\right]^{2}\right\}<\infty
$$

for all $k \geqslant 1$, then $L_{n}, n \geqslant 1$, defines a martingale for which

$$
E\left(L_{n}^{2}\right)=\sum_{k=1}^{n} \tau_{k}^{2} \quad \forall n \geqslant 1
$$

Proof. The first assertion follows directly from Lemma 1, induction, and division. That

$$
L_{n}=\sum_{k=1}^{n}\left(\frac{D g_{k, 1}}{g_{k}}\right)\left(X_{1}, \ldots, X_{k-1} ; X_{k}\right)
$$

is integrable for all $n$ follows directly from (5). For the martingale property,

$$
\begin{aligned}
E\left\{L_{k}-L_{k-1} \mid X_{1}=x_{1}, \ldots, X_{k-1}=x_{k-1}\right\} & =\int_{-\infty}^{\infty} D g_{k, 1}\left(x_{1}, \ldots, x_{k-1} ; x_{k}\right) \mathrm{d} x_{k} \\
& =D \bar{g}_{k, 1}\left(x_{1}, \ldots, x_{k-1}\right)=0
\end{aligned}
$$

for a.e. $x_{1}, \ldots, x_{k-1}$, by Lemma 3 , since $\bar{g}_{k}\left(x_{1}, \ldots, x_{k-1}\right)=1$ for all $x_{1}, \ldots, x_{k-1}$. The final assertion follows, since martingale differences are orthogonal.

Theorem 1. With the notation of the previous paragraph, suppose that

$$
H_{n} \stackrel{\mathrm{d}}{\rightarrow} \operatorname{Normal}\left(0, \alpha^{2}\right), \quad \exists \alpha>0,
$$

and

$$
\sup _{n} E\left\{\frac{1}{n} L_{n}^{2}\right\}<\infty
$$

Then

$$
\lim _{n} h_{n}(s)=\frac{1}{\alpha} \phi\left(\frac{s}{\alpha}\right)
$$

uniformly in $-\infty<s<\infty$, where $\phi$ denotes the standard normal density.

Proof. First observe that for all $s \in \mathbb{R}, t>0$ and $n \geqslant 1$,

$$
h_{n}(s+t)-h_{n}(s)=\int_{\left\{s<S_{n}^{*} \leqslant s+t\right\}} \frac{1}{\sqrt{n}} L_{n} \mathrm{~d} P,
$$


by Proposition 1. Since $\sup _{n}(1 / n) E\left(L_{n}^{2}\right)<\infty, L_{n} / \sqrt{n}, n \geqslant 1$, are uniformly integrable. Moreover, since $H_{n}$ is continuous for all $n$ and converges to a normal distribution as $n \rightarrow \infty$, it is easily seen that $\lim _{t \rightarrow 0+} \sup _{n \geqslant 1} H_{n}(s+t)-H_{n}(s)=0$ for all $s$. So, $h_{n}, n \geqslant 1$, are equicontinuous. That $h_{n}(s)$ converges to $\alpha^{-1} \dot{\phi}\left(\alpha^{-1} s\right)$ uniformly on compacts in $-\infty<s<\infty$ now follows from the main result of Boos (1985) and Sweeting (1986). So, it remains to show that $\lim _{|s| \rightarrow \infty} \sup _{n \geqslant 1} h_{n}(s)=0$. That $\lim _{s \rightarrow \infty} \sup _{n \geqslant 1} h_{n}(-s)$ $=0$ follows directly from (4), since $L_{n} / \sqrt{n}, n \geqslant 1$, are uniformly integrable and $\lim _{s \rightarrow-\infty} \sup _{n \geqslant 1} P\left\{S_{n}^{*} \leqslant\right.$ $-s\}=0 ;$ and $\lim _{s \rightarrow \infty} \sup _{n} h_{n}(s)=0$, by a similar argument.

Corollary 1. If $\sup _{k} \tau_{k}^{2}<\infty$ and (6) holds, then (8) holds.

Remark 1. The quantity $L_{n}$ is the score function (at $\theta=0$ ) for the statistical problem in which one observe $Y_{k}=X_{k}+\theta$ for $k=1, \ldots, n$ for some unknown $\theta \in \mathbb{R}$; and $E\left(L_{n}^{2}\right)$ is the Fisher information for this model. Thus, (7) requires that the Fisher information grow no faster than $n$.

\section{Stationary Markov processes}

In this section, $X_{1}, X_{2}, \ldots$, denotes a strictly stationary sequence with conditional densities $g_{k}, k \geqslant 1$, as in the previous section. Moreover, $X_{n}, n \geqslant 1$, is assumed to be a Markov chain of order $m-1$, where $2 \leqslant m<\infty$. That is, there are versions of $G_{k}, k \geqslant 1$, for which

$$
G_{k}\left(x_{1}, \ldots, x_{k-1} ; x\right)=G_{m}\left(x_{k-m+1}, \ldots, x_{k-1} ; x\right)
$$

for all $\left(x_{1}, x_{2}, \ldots\right) \in \mathbb{R}^{\infty}, x \in \mathbb{R}$ and $k \geqslant m$.

Corollary 2. If $g_{k}$ are almost differentiable in the direction 1 for all $k \leqslant m, \tau_{k}^{2}<\infty$ for all $k \leqslant m$, and (6) holds, then so does (8).

Proof. In this case $\tau_{k}^{2}=\tau_{m}^{2}$ for all $k>m$, so that $\sup _{k} \tau_{k}^{2}$ is finite.

In the remainder of this section, it is assumed that the densities $g_{k}, k \leqslant m$, are almost differentiable in all directions, and that $D g_{k, u}$ is of the form $D g_{k, u}=u \cdot \nabla g_{k}$ for all $\mathrm{u}$ for some essentially unique function $\nabla g_{k}$ for all $k \leqslant m$. Let

$$
\iota_{k}=E\left\{\left\|\frac{\nabla g_{k}}{g_{k}}\left(X_{1}, \ldots, X_{k-1} ; X_{k}\right)\right\|^{2}\right\}, \quad 1 \leqslant k \leqslant m,
$$

where $\|\cdot\|$ denotes the Euclidean norm, and observe that $\tau_{k}^{2} \leqslant k \iota_{k}$ for all $k=1, \ldots, m$.

Theorem 2. If $\iota_{m}<\infty$ and (6) holds, then (8) holds.

Proof. It suffices to show that $E\left(L_{m-1}^{2}\right)<\infty$, since then

$$
E\left(L_{n}^{2}\right)=E\left(L_{m-1}^{2}\right)+(n-m+1) \tau_{m}^{2} \leqslant E\left(L_{m-1}^{2}\right)+(n-m+1) m \iota_{m}=\mathrm{O}(n)
$$

as $n \rightarrow \infty$. To see this, first observe that

$$
f_{m-1}\left(x_{2}, \ldots, x_{m}\right)=\int_{-\infty}^{\infty} g_{m}\left(x_{1}, \ldots ; x_{m}\right) f_{m-1}\left(x_{1}, \ldots, x_{m-1}\right) \mathrm{d} x_{1}
$$


for a.e. $x_{2}, \ldots, x_{m}$. So, letting $\partial_{i}=e_{1} \cdot \nabla$, where $e_{t}=(0, \ldots, 1, \ldots, 0)$ denotes the $i$ th unit vector,

$$
\begin{aligned}
\partial_{i} f_{m-1}\left(x_{2}, \ldots, x_{m}\right)= & \int_{-\infty}^{\infty} \partial_{i} g_{m}\left(x_{1}, \ldots ; x_{m}\right) f_{m-1}\left(x_{1}, \ldots, x_{m-1}\right) \mathrm{d} x_{1} \\
& +\int_{-\infty}^{\infty} g_{m}\left(x_{1}, \ldots ; x_{m}\right) \partial_{i} f_{m-1}\left(x_{1}, \ldots, x_{m-1}\right) \mathrm{d} x_{1}
\end{aligned}
$$

for all $i=2, \ldots, m$ by Lemmas 1 and 3 . Here the second term is to be interpreted as zero when $i=m$. By Schwarz' inequality,

$$
\begin{aligned}
\left|\partial_{i} f_{m-1}\left(x_{2}, \ldots, x_{m}\right)\right| \leqslant & \sqrt{\int_{-\infty}^{\infty} \frac{\partial_{i} g_{m}\left(x_{1}, \ldots, ; x_{m}\right)^{2}}{g_{m}\left(x_{1}, \ldots, ; x_{m}\right)} f_{m-1}\left(x_{1}, \ldots, x_{m-1}\right) \mathrm{d} x_{1}} \\
& \times \sqrt{\int_{-\infty}^{\infty} g_{m}\left(x_{1}, \ldots, ; x_{m}\right) f_{m-1}\left(x_{1}, \ldots, x_{m-1}\right) \mathrm{d} x_{1}} \\
& +\sqrt{\int_{-\infty}^{\infty} g_{m}\left(x_{1}, \ldots, ; x_{m}\right) \frac{\partial_{i} f_{m-1}\left(x_{1}, \ldots, x_{m-1}\right)^{2}}{f_{m-1}\left(x_{1}, \ldots, x_{m-1}\right)} \mathrm{d} x_{1}} \\
& \times \sqrt{\int_{-\infty}^{\infty} g_{m}\left(x_{1}, \ldots, x_{m}\right) f_{m-1}\left(x_{1}, \ldots, x_{m-1}\right) \mathrm{d} x_{1}}
\end{aligned}
$$

for a.e. $x_{2}, \ldots, x_{m}$. So, since the second and fourth integrals are just $f_{m-1}\left(x_{2}, \ldots, x_{m}\right)$ and $g_{m}$ is a conditional density,

$$
\begin{aligned}
\int & \cdots \int_{\mathbb{R}^{m-1}} \frac{\partial_{i} f_{m-1}\left(x_{2}, \ldots x_{m}\right)^{2}}{f_{m-1}\left(x_{2}, \ldots x_{m}\right)} \mathrm{d} x_{2} \cdots \mathrm{d} x_{m} \\
\leqslant & 2 \int \cdots \int_{\mathbb{R}^{m}} \frac{\partial_{i} g_{m}\left(x_{1}, \ldots ; x_{m}\right)^{2}}{g_{m}\left(x_{1}, \ldots ; x_{m}\right)} f_{m-1}\left(x_{1}, \ldots, x_{m-1}\right) \mathrm{d} x_{1} \cdots \mathrm{d} x_{m} \\
& +2 \int \cdots \int_{\mathbb{R}^{m}} g_{m}\left(x_{1}, \ldots ; x_{m}\right) \frac{\partial_{i} f_{m-1}\left(x_{1}, \ldots, x_{m-1}\right)^{2}}{f_{m-1}\left(x_{1}, \ldots, x_{m-1}\right)} \mathrm{d} x_{m} \cdots \mathrm{d} x_{1} \\
\leqslant & 2 \iota_{m}+2 \int \cdots \int_{\mathbb{R}^{m-1}} \frac{\partial_{i} f_{m-1}\left(x_{1}, \ldots, x_{m-1}\right)^{2}}{f_{m-1}\left(x_{1}, \ldots, x_{m-1}\right)} \mathrm{d} x_{1} \cdots \mathrm{d} x_{m-1}
\end{aligned}
$$

for all $i=2, \ldots, m$. When $i=m$, the last integral is absent, so that the first is finite. Then, when $i=m-1$, the last integral is finite, so that the first is finite too. By induction the first integral is finite for all $i=2, \ldots, m$, thus proving the proposition.

Remark 2. There are potential relationships between (6) and the condition that $\iota_{m}$ be finite. Using Boos' theorem again, it may be shown that if $\iota_{m}<\infty$ and $X_{1}, X_{2}, \ldots$, is mixing, then $X_{1}, X_{2}, \ldots$, is strongly (uniformly) mixing, one of a set of conditions which imply asymptotic normality.

\section{References}

Boos, D. (1985), A converse to Scheffe's theorem, Ann. Statist. $13,423-427$.
Hall, P. and C. Heyde (1980), Martingale Limit Theory and its Application (Academic Press, New York). 
Jeganathan, P. (1987), Strong convergence of the distributions of estimators, Ann. Statist. 15, 1699-1708.

Klassen, C. (1984), Location estimators and spread, Ann. Statist. 12, 311-321.

Lalley, S. (1986), Ruelle's Perron Frobenius theorem and the central limit theorem for additive functionals of Gibb's states, in: J. Van Ryzin, ed., Adaptive Procedures and Related Topics (Inst. Math. Statist., Hayward, CA) pp. 428-446.
Peligrad, M. (1986), Recent advances in the central limit theorem and its weak invariance principle for mixing sequences of random variables (a survey), in: E. Eberlein and M.S. Taqqu, eds., Dependence in Probability and Statistics (Birkhauser, Basel).

Stein, C. (1981), Estimation of the mean of a multivariate normal distribution, Ann. Statist. 9, 1135-1152.

Sweeting, T. (1986), A converse to Scheffe's theorem, Ann. Statist. 14, 1252-1256. 